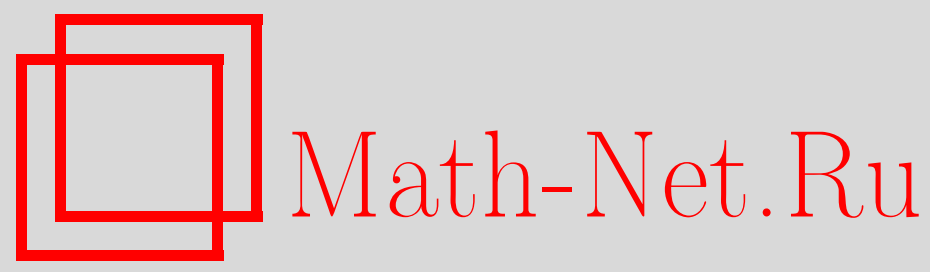

А. Л. Якымив, Некоторые свойства субэкспоненциальных распределений, Матем. заметки, 1997, том 62, выпуск 1, 138-144

DOI: https://doi.org/10.4213/mzm1597

Использование Общероссийского математического портала Math-Net.Ru подразумевает, что вы прочитали и согласны с пользовательским соглашением http://www.mathnet.ru/rus/agreement

Параметры загрузки:

IP: 54.162 .27 .143

26 апреля 2023 г., 13:57:51 


\section{НЕКОТОРЫЕ СВОЙСТВА \\ СУБЭКСПОНЕНЦИАЛЬНЫХ РАСПРЕДЕЛЕНИЙ}

\section{А. Л. Якымив}

Неотрицательная случайная величина $X$ имеет субэкспоненциальное распределение, если $(1-G(t)) /(1-F(t)) \rightarrow 2$ при $t \rightarrow \infty$, где $F(t)=\mathrm{P}\{X \leqslant t\}$, a $G(t)-$ свертка $F(t)$ с собой. Даны условия на распределения независимых неотрицательных случайных величин $X$ и $Y$, при выполнении которых $\max (X, Y)$ и $\min (X, Y)$ имеют субэкспоненциальное распределение.

Библиография: 10 названий.

Мы будем говорить, что распределение неотрицательной случайной величины $X$ cyбәкспоненииально, и писать $X \in S$, если существует

$$
\lim _{t \rightarrow \infty} \frac{1-F^{2 *}(t)}{1-F(t)}=2,
$$

где $F(t)$ - функция распределения, $X, F^{2 *}(t)$ - свертка $F(t)$ с собой: $F(t)=\mathrm{P}\{X \leqslant t\}$,

$$
F^{2 *}(t)=\int_{0}^{t} F(t-u) d F(u), \quad t \geqslant 0 .
$$

Субэкспоненциальные распределения были введены в 1964 году В.П. Чистяковым [1] и в дальнейшем получили приложения в теории ветвящихся процессов, теории массового обслуживания, теории восстановления, теории безгранично делимых распределений (см. [2]-[9]).

В настоящей заметке изучаются вопросы, которые еще не были предметом рассмотрения ранее. А именно, пусть случайные величины $X$ и $Y$ независимы, $X, Y \in S$. В каком случае $\max (X, Y)$ и $\min (X, Y) \in S ?$ В теореме 1 вопрос о принадлежности $\max (X, Y)$ множеству $S$ сводится к уже изучавшейся ранее проблемепринадлежности суммы $X+Y$ множеству субэкспоненциальных распределений (см. статьи [5]-[7], [9], [10]). А именно, показано, что $\max (X, Y) \in S$ тогда и только тогда, когда $X+Y \in S$. Более сложньм для изучения оказался вопрос о принадлежности $\min (X, Y)$ множеству $S$. Здесь даны только достаточные условия (теорема 2). В заключение в теореме 3 даны достаточные условия принадлежности $\max (X, Y)$ множеству $S$. Перейдем к формулировке полученных результатов.

Положим $G(t)=\mathrm{P}\{Y \leqslant t\}, T(t)=1-F(t), H(t)=1-G(t), t \geqslant 0$. Мы будем писать, что $X \in D$, если

$$
\varlimsup_{t \rightarrow \infty} \frac{T(t)}{T(2 t)}<\infty .
$$

Если вьполнено (1), то функция $T(t)$ назьвается мажсорируемо меняющейся на бесконечности. 
Tеорема 1. Пусть $X$ и $Y$ независимы, $X, Y \in S . \max (X, Y) \in S$ тогда и только тогда, когда $X+Y \in S$.

Tеорема 2. Пусть $X$ и $Y$ независимы, $X, Y \in S$. Если $X \in D$ и $Y \in D$, то $\min (X, Y) \in S$.

Пусть $G(t)=a_{1} G_{1}(t)+a_{2} G_{2}(t)+a_{3} G_{3}(t), t \geqslant 0$, где $a_{i} \geqslant 0, i=1,2,3, a_{1}+a_{2}+a_{3}=1$, $G_{1}(t), G_{2}(t), G_{3}(t)$ - соответственно дискретная, абсолютно непрерывная и сингулярная функции распределения, $H_{i}(t)=1-G_{i}(t), t \geqslant 0, i=1,2,3$. Предположим, что все точки роста $G_{1}(t)$ расположены в последовательности $x_{1}<x_{2}<\cdots$ и

$$
G_{1}\left(x_{n}\right)-G_{1}\left(x_{n}-\right)=p_{n}, \quad n \in \mathbb{N}, \quad \sum_{n \in \mathbb{N}} p_{n}=1 .
$$

Теорема 3. Пусть $X$ и $Y$ независимы, $X, Y \in S, \mathrm{M} X<\infty, X \in D$ и выполнены следуюшие предположения:

1) для некоторого $\mu>0$ и всех $n \in \mathbb{N} x_{n+1}-x_{n} \leqslant \mu$ и при $n \rightarrow \infty$

$$
p_{n}=o\left(T\left(x_{n}\right)\right)
$$

2) плотность $g(t)$ функиии распределения $G_{2}(t)$ удовлетворяет соотношению

$$
g(t)=o(T(t)) \quad(t \rightarrow \infty)
$$

3) прut $\rightarrow \infty$

$$
H_{3}(t)=o(T(t))
$$

Тогда $\max (X, Y) \in S$.

Для доказательства теорем 1-3 нам понадобятся следующие три леммы.

Лемма 1 (см. [10]). Пусть $X \in S$. Тогда существует функиия $\varphi(t) \in[0, t]$ при $t \geqslant 0$ такая, что при $t \rightarrow \infty$

$$
\varphi(t) \rightarrow+\infty, \quad \varphi(t)=o(t), \quad \frac{T(t-\varphi(t))}{T(t)} \rightarrow 1
$$

Лемма 2. Пусть $X \in S$. Тогда для произвольной функиии $\varphi(t)$, удовлетворяющей (4), выполнено следующее соотношение:

$$
\int_{\varphi(t)}^{t} T(t-u) d F(u)=o(T(t)) \quad(t \rightarrow \infty)
$$

Лемма 3. Пусть $X$ и $Y$ независимь $, X, Y \in S$. Зафиксируем произвольную функиию $\varphi(t)$, удовлетворяюш,ую (4). Тогда $X+Y \in S$ тогда и только тогда, $\kappa о г д а$

$$
\int_{\varphi(t)}^{t} T(t-u) d G(u)=o(T(t)+H(t)) \quad(t \rightarrow \infty) .
$$

Доказательство леммы 2 несложно и здесь не приводится. Лемма 3 следует из леммы 1 работы [10] и соотношений (4) и (5) той же работы. 
ДОКАЗАТЕЛЬСТВО ТЕОРЕМЫ 1 . Положим при $t \geqslant 0 U(t)=F(t) G(t), V(t)=1-U(t)$. Пусть $X, Y \in S$ и $Z_{1}$ и $Z_{2}$ - независимые случайные величины с функцией распределения $U(t)$. Заметим, что

$$
\mathrm{P}\left\{Z_{1}+Z_{2}>t\right\}=1-\int_{0}^{t} U(t-u) d U(u)=V(t)+\int_{0}^{t} V(t-u) d U(u) .
$$

Поэтому для доказательства теоремы 1 достаточно показать, что $X+Y \in S$ тогда и только тогда, когда при $t \rightarrow \infty$

$$
I=\int_{0}^{t} V(t-u) d U(u) \sim V(t) .
$$

Подставляя в (5) $V(t)=T(t)+H(t)-T(t) H(t)$, разобьем интеграл $I$ на три части: $I=I_{1}+I_{2}-I_{3}$. Для $I_{1}$ имеем

$$
\begin{aligned}
I_{1} & =\int_{0}^{t} T(t-u) d(F(u) G(u)) \\
& =\int_{0}^{t} T(t-u) F(u) d G(u)+\int_{0}^{t} T(t-u) G(u) d F(u)=J_{1}+J_{2} .
\end{aligned}
$$

Рассмотрим сначала $J_{2}$. Для функции $\varphi(t)$, удовлетворяющей условиям (4), имеем

$$
J_{2}=\int_{0}^{\varphi(t)} T(t-u) G(u) d F(u)+\int_{\varphi(t)}^{t} T(t-u) G(u) d F(u) .
$$

В силу леммы 1 при $t \rightarrow \infty$

$$
\int_{0}^{\varphi(t)} T(t-u) G(u) d F(u) \sim T(t) \int_{0}^{\infty} G(u) d F(u) .
$$

Согласно лемме 2

$$
\int_{\varphi(t)}^{t} T(t-u) G(u) d F(u) \leqslant \int_{\varphi(t)}^{t} T(t-u) d F(u)=o(T(t))
$$

при $t \rightarrow \infty$. Поэтому

$$
J_{2}=(1+o(1)) T(t) \int_{0}^{\infty} G(u) d F(u) \quad(t \rightarrow \infty) .
$$

Далее,

$$
J_{1}=\int_{0}^{\varphi(t)} T(t-u) F(u) d G(u)+\int_{\varphi(t)}^{t} T(t-u) F(u) d G(u) .
$$

Точно так же, как и ранее, устанавливаем, что

$$
\int_{0}^{\varphi(t)} T(t-u) F(u) d G(u)=(1+o(1)) T(t) \int_{0}^{\infty} F(u) d G(u)
$$


при $t \rightarrow \infty$. Поэтому

$$
J_{1}=(1+o(1)) T(t) \int_{0}^{\infty} F(u) d G(u)+(1+o(1)) \int_{\varphi(t)}^{t} T(t-u) d G(u)
$$

при $t \rightarrow \infty$. Из (6)-(8) следует, что

$$
I_{1}=(1+o(1)) T(t)+(1+o(1)) \int_{\varphi(t)}^{t} T(t-u) d G(u)
$$

Пусть $\psi(t)$ - функция, обладающая всеми свойствами функции $\varphi(t)$, только относительно $H(t)$. Повторяя рассуждения, применявшиеся при доказательстве $(9)$, имеем

$$
I_{2}=(1+o(1)) H(t)+(1+o(1)) \int_{\psi(t)}^{t} H(t-u) d F(u) .
$$

Положим $\mu(t)=\min (\varphi(t), \psi(t))$. В силу леммы 1

$$
\int_{0}^{\mu(t)} T(t-u) H(t-u) d U(u) \sim T(t) H(t)=o(T(t)+H(t))
$$

при $t \rightarrow \infty$. Далее, согласно лемме 2

$$
\begin{aligned}
\int_{\mu(t)}^{t} T(t-u) H(t-u) d U(u)= & \int_{\mu(t)}^{t} T(t-u) H(t-u) F(u) d G(u) \\
& +\int_{\mu(t)}^{t} T(t-u) H(t-u) G(u) d F(u) \\
\leqslant & \int_{\mu(t)}^{t} H(t-u) d G(u)+\int_{\mu(t)}^{t} T(t-u) d F(u) \\
= & o(H(t))+o(T(t))=o(H(t)+T(t))
\end{aligned}
$$

при $t \rightarrow \infty$, так как функция $\mu(t)$ обладает всеми свойствами функций $\varphi(t)$ и $\psi(t)$ относительно $T(t)$ и $H(t)$. Поэтому

$$
I_{3}=\int_{0}^{t} T(t-u) H(t-u) d U(u)=o(H(t)+T(t)) \quad(t \rightarrow \infty) .
$$

Из (10)-(12) следует, что при $t \rightarrow \infty$

$$
I=(1+o(1)) V(t)+(1+o(1))\left(\int_{\varphi(t)}^{t} T(t-u) d G(u)+\int_{\psi(t)}^{t} H(t-u) d F(u)\right) .
$$

Пусть $X+Y \in S$. Тогда соотношение (13) и лемма 3 влекут (5). Если $\max (X, Y) \in S$, то из (5) и (13) вьводим, что

$$
\int_{\varphi(t)}^{t} T(t-u) d G(u)=o(T(t)+H(t)) \quad(t \rightarrow \infty),
$$

откуда при помощи леммы 3 следует, что $X+Y \in S$. Теорема 1 доказана. 
ДОКАЗАТЕЛЬСТВО ТЕОРЕМЫ 2. ПоЛОЖим

$$
V(t)=\mathrm{P}\{\min (X, Y)>t\}=T(t) H(t), \quad U(t)=1-V(t), \quad t \geqslant 0 .
$$

Как и в теореме 1 , докажем, что

$$
\int_{0}^{t} V(t-u) d U(u) \sim V(t) \quad(t \rightarrow \infty) .
$$

Согласно лемме 1 мы можем выбрать функцию $\varphi(t) \in[0, t]$ такую, что $\varphi(t)=o(t)$ и $T(t-\varphi(t)) \sim T(t), H(t-\varphi(t)) \sim H(t)$ при $t \rightarrow \infty$. Прежде всего заметим, что в силу выписанных свойств функции $\varphi(t)$

$$
\int_{0}^{\varphi(t)} V(t-u) d U(u) \sim V(t) \quad(t \rightarrow \infty) .
$$

Поскольку $H(t)$ и $T(t)$ мажорируемо меняются на бесконечности, то

$$
\begin{aligned}
\int_{\varphi(t)}^{t / 2} V(t-u) T(u) d G(u) & \leqslant V\left(\frac{t}{2}\right) \int_{\varphi(t)}^{t} T(u) d G(u) \\
& \leqslant V\left(\frac{t}{2}\right) T(\varphi(t)) H(\varphi(t))=o(V(t)) \quad(t \rightarrow \infty) .
\end{aligned}
$$

Далее, по той же причине

$$
\begin{aligned}
\int_{t / 2}^{t-\varphi(t)} V(t-u) T(u) d G(u) & \leqslant V(\varphi(t)) \int_{t / 2}^{t} T(u) d G(u) \\
& \leqslant V(\varphi(t)) T\left(\frac{t}{2}\right) H\left(\frac{t}{2}\right)=o(V(t)) \quad(t \rightarrow \infty) .
\end{aligned}
$$

Заметим также, что

$$
\begin{aligned}
\int_{t-\varphi(t)}^{t} V(t-u) T(u) d G(u) & \leqslant T(t-\varphi(t))(H(t-\varphi(t))-H(t)) \\
& =O(T(t)) o(H(t))=o(V(t)) \quad(t \rightarrow \infty) .
\end{aligned}
$$

Из соотношений (16)-(18) следует, что

$$
\int_{\varphi(t)}^{t} V(t-u) T(u) d G(u)=o(V(t)) \quad(t \rightarrow \infty) .
$$

Точно так же устанавливается, что

$$
\int_{\varphi(t)}^{t} V(t-u) H(u) d F(u)=o(V(t)) \quad(t \rightarrow \infty) .
$$

Из (19) и (20) получаем, что

$$
\int_{\varphi(t)}^{t} V(t-u) d U(u)=o(V(t)) \quad(t \rightarrow \infty) .
$$

Из (15) и (21) следует (14). Теорема доказана. 
ДоКАЗАТЕЛЬСТВО ТЕОРЕМЫ 3. Пусть функция $\varphi(t)$ удовлетворяет условиям (4). Из соотношения (6) работы [10] следует, что для любого фиксированного $\lambda \in(0,1)$ при $t \rightarrow \infty$

$$
\int_{\varphi(t)}^{\lambda t} T(t-y) d G(y)=o(T(t))
$$

Поскольку $T(t)$ мажорируемо меняется на бесконечности, то в силу (3) при $t \rightarrow \infty$

$$
\int_{\lambda t}^{t} T(t-y) d G_{3}(y) \leqslant H_{3}(\lambda t)=o(T(\lambda t))=o(T(t)) .
$$

Точно так же, как и при доказательстве теоремы 1 из [10], устанавливаем, что

$$
\int_{\lambda t}^{t} T(t-y) d G_{2}(y)=o(T(t)) \quad(t \rightarrow \infty) .
$$

Далее,

$$
\int_{\lambda t}^{t} T(t-y) d G_{1}(y)=\sum T\left(t-x_{n}\right) p_{n} \leqslant \sup p_{n} \sum T\left(t-x_{n}\right),
$$

где сумма и супремум берутся по всем тем натуральным $n$, для которых $x_{n} \in[\lambda t, t]$. Заметим, что

$$
\sum T\left(t-x_{n}\right) \leqslant \sum_{m=0}^{[t \nu]} T(\mu m) \leqslant 1+\frac{1}{\mu} \int_{0}^{t(1-\lambda)} T(y) d y \leqslant 1+\frac{\mathrm{M} X}{\mu}
$$

где условия суммирования в первой сумме те же, что и ранее, $\nu=(1-\lambda) / \mu$ (мы воспользовались неравенством

$$
T(\mu m) \leqslant \frac{1}{\mu} \int_{\mu(m-1)}^{\mu m} T(y) d y
$$

справедливьм при $m \geqslant 1)$. Зафиксируем произвольное $\varepsilon>0$. Согласно $(2)$ для него найдется такое $b>0$, что при $x_{n} \geqslant b$

$$
p_{n} \leqslant \varepsilon T\left(x_{n}\right) .
$$

Из неравенств (24)-(26) следует, что при $t \geqslant b / \lambda$

$$
\int_{\lambda t}^{t} T(t-y) d G_{1}(y) \leqslant \varepsilon T(\lambda t)\left(1+\frac{\mathrm{M} X}{\mu}\right) .
$$

Из (27) получаем, что

$$
\varlimsup_{t \rightarrow \infty} \int_{\lambda t}^{t} T(t-y) \frac{d G_{1}(y)}{T(t)} \leqslant C \varepsilon,
$$

где

$$
C=\left(1+\frac{\mathrm{M} X}{\mu}\right) \varlimsup_{t \rightarrow \infty} \frac{T(\lambda t)}{T(t)}<\infty,
$$


так как $T(t)$ мажорируемо меняется на бесконечности. В силу произвольности $\varepsilon$ из $(28)$ следует, что

$$
\int_{\lambda t}^{t} T(t-y) d G_{1}(y)=o(T(t)) \quad(t \rightarrow \infty) .
$$

В итоге из $(22),(23)$ и (29) получаем, что

$$
\int_{\lambda t}^{t} T(t-y) d G(y)=o(T(t)) \quad(t \rightarrow \infty) .
$$

Таким образом, при $t \rightarrow \infty$

$$
\int_{\varphi(t)}^{t} T(t-y) d G(y)=o(T(t))
$$

Из последнего соотношения, теоремы 1 и леммы 3 получаем требуемое. Теорема доказана.

\section{СПИСОК ЦИТИРОВАННОЙ ЛИТЕРАТУРЫ}

[1] Чистяков В. П. Теорема о суммах независимых положительных случайных величин и ее приложения к ветвящимся случайным процессам // Теор. вероятностей и ее применения. 1964. T. 9. №4. C. 710-718.

[2] Сгибнев М.C. Асимптотика безгранично делимых распределений в $\mathbb{R} / /$ Сиб. матем. ж. 1990. T. 31. № 1. C. 135-140.

[3] Chover J., Ney P., Wainger S. Degeneracy properties of subcritical branching processes // Ann. Probab. 1973. V. 1. P. 663-673.

[4] Teugels J. L. The class of subexponential distributions // Ann. Probab. 1975. V. 3. P. 1000-1011.

[5] Goldie C. M. Subexponential distributions and dominated-variation tails // J. Appl. Probab. 1978. V. 15. P. 440-442.

[6] Embrechts P., Goldie C. M. On closure and factorisation properties of subexponential and related distributions // J. Austral. Math. Soc. 1980. V. 29. P. 243-256.

[7] Cline D. B. H. Convolution of distributions with exponential and subexponential tails // J. Austral. Math. Soc. 1987. V. 93. P. 347-365.

[8] Kluppelberg C. Subexponential distributions and integrated tails // J. Appl. Probab. 1988. V. 25. P. 132-141.

[9] Leslie J. R. On the non-closure under convolution of the subexponential family // J. Appl. Probab. 1989. V. 26. P. 58-66.

[10] Якымив А. Л. Достаточные условия субэкспоненциальности свертки двух распределений // Матем. заметки. 1995. Т. 58. № 5. С. 778-781. 